\title{
Corrigendum
}

\section{Laboratory-size X-ray Microscope using Wolter Mirror Optics and an Electron-impact X-ray Source for Multi-energy Observation - CORRIGENDUM}

Akira Ohba, Tomoyasu Nakano, Shinobu Onoda, Takahiro Mochizuki, Katsuhiro Nakamoto, Hisaya Hotaka, Katsuyoshi Fujita, Shinji Ohsuka, Motosuke Miyoshi, Keita Soda and Takao Hamakubo

doi: https://doi.org/10.1017/S1431927618013429, Published by Cambridge University Press, 10 August 2018

In the original publication, author Motosuke Miyoshi's name was misspelled. The original has since been corrected.

\section{Reference}

Ohba A, Nakano T, Onoda S, Mochizuki T, Nakamoto K, Hotaka H, Fujita K, Ohsuka S, Miyoshi M, Soda K and Hamakubo T (2018) Laboratory-size x-ray microscope using Wolter mirror optics and an electron-impact x-ray source for multi-energy observation. Microsc Microanal 24(S2), 214-215. doi 10.1017/ S1431927618013429. 